\title{
Hyperbaric oxygen therapy ameliorates acute brain injury after porcine intracerebral hemorrhage at high altitude
}

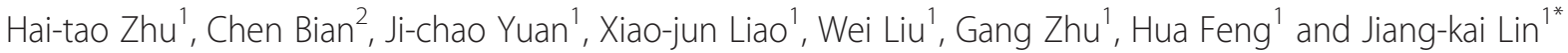

\begin{abstract}
Introduction: Intracerebral hemorrhage $(\mathrm{ICH})$ at high altitude is not well understood to date. This study investigates the effects of high altitude on $\mathrm{ICH}$, and examines the acute neuroprotection of hyperbaric oxygen (HBO) therapy against high-altitude $\mathrm{ICH}$.
\end{abstract}

Methods: Minipigs were placed in a hypobaric chamber for $72 \mathrm{~h}$ before the operation. ICH was induced by an infusion of autologous arterial blood $(3 \mathrm{ml})$ into the right basal ganglia. Animals in the high-altitude $\mathrm{ICH}$ group received HBO therapy (2.5 ATA for $60 \mathrm{~min}$ ) $30 \mathrm{~min}$ after $\mathrm{ICH}$. Blood gas, blood glucose and brain tissue oxygen partial pressure $\left(\mathrm{PbtO}_{2}\right)$ were monitored continuously for animals from all groups, as were microdialysis products including glucose, lactate, pyruvate and glutamate in perihematomal tissue from 3 to $12 \mathrm{~h}$ post- $\mathrm{ICH}$.

Results: High-altitude ICH animals showed significantly lower $\mathrm{PbtO}_{2}$, higher lactate/pyruvate ratio (LPR) and glutamate levels than low-altitude ICH animals. More severe neurological deficits, brain edema and neuronal damage were also observed in high-altitude ICH. After $\mathrm{HBO}$ therapy, $\mathrm{PbtO}_{2}$ was significantly increased and LPR and glutamate levels were significantly decreased. Brain edema, neurological deficits and neuronal damage were also ameliorated.

Conclusions: The data suggested a more serious disturbance of tissue oxygenation and cerebral metabolism in the acute stage after ICH at high altitude. Early HBO treatment reduced acute brain injury, perhaps through a mechanism involving the amelioration of the derangement of cerebral oxygenation and metabolism following high-altitude $\mathrm{ICH}$.

\section{Introduction}

Intracerebral hemorrhage $(\mathrm{ICH})$ is the second most common and the deadliest subtype of stroke, with a high mortality rate [1]. It represents between 10 and $15 \%$ of all strokes that occur in the US, Europe and Australia, and between 20 and $30 \%$ of all strokes that occur in Asian countries [2]. The 30-day mortality rate is approximately $40 \%$. Furthermore, $\mathrm{ICH}$ has a significant morbidity among individuals who survive, and only $20 \%$ of survivors are independent at six months post-stroke.

A growing number of people travel to, or reside at altitudes higher than 2,500 m, such as the North American Rocky Mountains and Chinese Qinghai-Tibetan Plateau,

\footnotetext{
* Correspondence: jklin@tmmu.edu.cn

${ }^{1}$ Department of Neurosurgery, Southwest Hospital, Third Military Medical University, 30 Gaotanyan Street, Chongqing 400038, China

Full list of author information is available at the end of the article
}

due to social and economic development. About 140 million people live permanently in high-altitude regions and their health needs more public awareness. Acute mountain sickness, high-altitude cerebral edema and high-altitude pulmonary edema comprise the main components of high-altitude illnesses. However, less well known are the other conditions, chiefly neurological, that may arise completely outside the usual definition of altitude sickness [3]. Stroke is outside the focus of the majority, and remains a major disease that seriously threatens the health of the people living at high altitudes. Some of the few studies on this subject have reported that long-term residence at high altitudes was associated with a higher risk of stroke, almost 10 times greater than residence at low altitude $[4,5]$. Moreover, stroke at a younger age occurs more frequently at high altitude than at low altitude [6]. Another study showed ICH to be the 
dominant subtype of high-altitude stroke, and it induced higher disability and mortality rates than at low altitude [7]. The etiology and clinical manifestations of high-altitude $\mathrm{ICH}$ are quite different from those of low-altitude ICH. Thus far, we know little about secondary brain injury and the pathophysiology related to high-altitude $\mathrm{ICH}$; thus, effective therapy for this disease is also very limited.

Several cerebral monitoring techniques permit continuous monitoring of cerebral physiology, such as brain tissue oxygen partial pressure $\left(\mathrm{PbtO}_{2}\right)$ and neurochemistry $[8,9]$. Use of these modalities is beneficial to optimize brain oxygen utilization and metabolism in patients with acute brain injury. It may provide an extended window for the prevention, early detection and treatment of ongoing secondary neuronal injury, and help to improve outcomes after ICH $[10,11]$. However, the changes in cerebral oxygenation and metabolism in the early stage of high-altitude $\mathrm{ICH}$, and the differences between high-altitude and low-altitude ICH regarding these parameters are unknown.

Early hyperbaric oxygen (HBO) therapy mitigates blood-brain barrier disruption and facilitates angiogenesis after $\mathrm{ICH}$. HBO therapy also reduces secondary hemorrhage after focal cerebral ischemia [12]. The amount of oxygen that is dissolved in plasma and tissues can be elevated dramatically via raising the pressure and oxygen content of inspired air. This may improve the aerobic and neurochemical milieu in the injured brain region [13]. Hypobaric hypoxia, a prime characteristic of high-altitude areas, is a risk factor for high-altitude stroke [5, 14]. Thus, treatment of high-altitude-induced hypoxemia using $\mathrm{HBO}$ is logical and necessary. However, the effects of $\mathrm{HBO}$ on acute brain injury induced by high-altitude ICH have not been reported. Currently the underlying mechanisms of $\mathrm{HBO}$ treatment, especially cerebral oxygenation and metabolism, are not well understood.

Previously, we established a porcine model of highaltitude $\mathrm{ICH}$, and observed more severe tissue lesion and neurological deficit seven days after ICH at high altitude compared to low altitude [15]. In this study, one goal was to evaluate the differences between low-altitude and high-altitude ICH with regards to cerebral oxygenation and metabolism in the acute stage (within $12 \mathrm{~h}$ ) of porcine ICH. The other goal was to evaluate the effects of early treatment with $\mathrm{HBO}$ on brain damage in highaltitude $\mathrm{ICH}$ via multimodality monitoring.

\section{Methods}

\section{Animals}

Forty-two male Guizhou Congjiang minipig (China) (15 to $20 \mathrm{~kg}$ ) were obtained from the Experimental Animal Center of the Third Military Medical University,
Chongqing, China. All experiments were conducted in accordance with animal care guidelines approved by the Animal Ethics Committee of the Third Military Medical University. The animals were housed with a 12-hour light/dark cycle and water and food provided ad libitum. They were randomly divided into five groups: plain (low-altitude) sham operation group (PS, six animals), plain blood-infusion group (PI, 10 animals), highaltitude sham operation group (HS, six animals), highaltitude blood-infusion group (HI, 10 animals) and high-altitude blood-infusion plus $\mathrm{HBO}$ therapy group (HI-HBO, 10 animals). PI, HI and HI-HBO animals received autologous arterial blood infusions; PS and HS animals received the same surgery but no blood infusions. The schematic representation of experimental timeline is shown in Fig. 1a.

\section{Animal surgical preparation}

Pigs were initially anesthetized with ketamine $(25 \mathrm{mg} / \mathrm{kg}$ Hengrui, Jiangsu, China) administered intramuscularly. After sedation, pentobarbital (30 mg/kg Xinya, Shanghai, China) was administered through an ear vein to achieve a deep surgical level of anesthesia. After placement of the femoral vein catheter, pentobarbital was infused at a rate of $10 \mathrm{mg} / \mathrm{kg}$ per hour until $12 \mathrm{~h}$ after $\mathrm{ICH}$. Core temperature was measured with a rectal thermistor probe (Daxiong, Shenzhen, China). The right femoral artery was catheterized for mean arterial pressure (MAP) monitoring, and to permit the withdrawal of blood samples for determination of respiratory gases, acid-base status and glucose concentrations every three hours (Table 1).

\section{Intracerebral blood infusion}

Surgery was performed as described previously, with moderate modifications $[15,16]$. Each animal's head was shaved and disinfected, and fully aseptic techniques were used. Three burr holes ( $2 \mathrm{~mm}$ in diameter) were drilled in the cranium: hole A (10 mm right, $2 \mathrm{~mm}$ posterior, for blood infusion), hole $\mathrm{B}$ ( $8 \mathrm{~mm}$ right, $8 \mathrm{~mm}$ posterior, for $\mathrm{PbtO}_{2}$ monitoring) and hole $\mathrm{C}(16 \mathrm{~mm}$ right, $8 \mathrm{~mm}$ posterior, for MD monitoring) (Fig. 1b). The right was relative to the sagittal suture, and the posterior was relative to the coronal suture. A 22-gauge sterile intravenous catheter (0.8 $\mathrm{mm}$ diameter, $25 \mathrm{~mm}$ long, Jierui, Weihai, China) was placed stereotaxically into the right basal ganglia $(25 \mathrm{~mm}$ below the skull) and fixed firmly to the skull. Then, blood $(1 \mathrm{ml})$ extracted from the right femoral artery was injected slowly into brain tissue through the inner cannula. A rate of $0.2 \mathrm{ml} / \mathrm{min}$ was controlled using a high-pressure syringe pump (Longerpump, Hebei, China). After blood infusion, the needle core was inserted into the inner cannula and maintained for $10 \mathrm{~min}$. Next, the needle core was extracted and another $2 \mathrm{ml}$ blood was injected slowly at the 


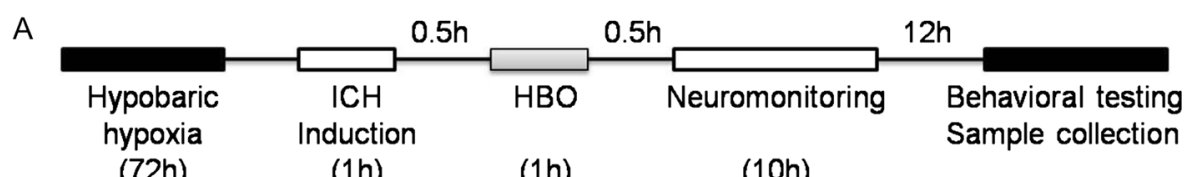

(72h) $\quad(1 \mathrm{~h})$

$(1 \mathrm{~h})$

(10h)
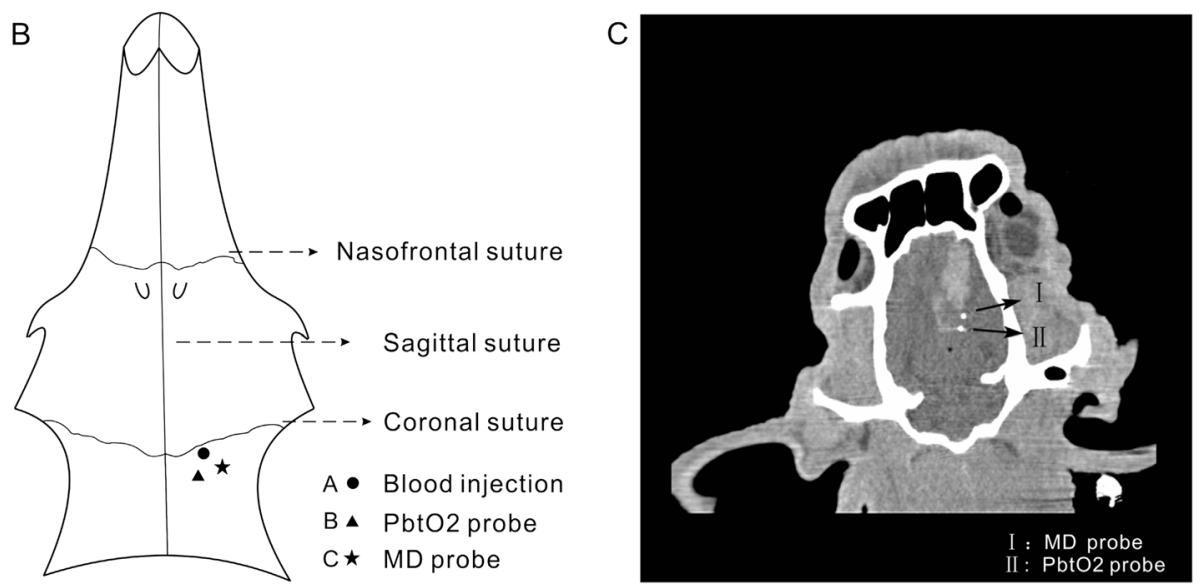

Fig. 1 The $\mathrm{ICH}$ induction and the positioning of the probes. a The schematic representation of the experimental timeline. $\mathbf{b}$ Schematic drawing of catheter positioning relative to the injection site of the hematoma in the minipig cranium. c The relative position of the $\mathrm{PbtO} \mathrm{O}_{2}$ probe and $\mathrm{MD}$ probe in perihematomal tissue were confirmed using computed tomography

same rate. The needle core then was reinserted and maintained for $20 \mathrm{~min}$, before the skull holes were sealed with bone wax.

\section{Experimental hypobaric hypoxia}

To simulate hypobaric hypoxia, a hypobaric chamber (Moon, Yantai, China) was used. HS, HI and HI-HBO animals were placed in the temperature-controlled hypobaric chamber $\left(20 \pm 1{ }^{\circ} \mathrm{C}\right)$ for $72 \mathrm{~h}$; the normal air in the chamber was adjusted to 0.6 ATA pressure, $14.5 \%$ oxygen content (simulating an altitude of 4,000 m). The chamber was opened twice a day (for approximately 30 min each day) for feeding and cleaning. We slowly increased the air pressure in the chamber until it reached the normal atmospheric pressure of our laboratory (about $15 \mathrm{~min}$ ) before opening the door of the chamber. After feeding and cleaning, we closed the door and then slowly decreased the air pressure in the chamber until the pressure was stabilized at 0.6 ATA (also about $15 \mathrm{~min})$. Three days later, the minipigs received the same surgery as the PI group described above, and were kept in the hypobaric chamber with 0.6 ATA after ICH. During the entire experiment, an investigator was present to observe the animals in the chamber.

\section{Hyperbaric oxygen therapy plan}

A hyperbaric pure oxygen chamber (Moon, Yantai, China) was used. HI-HBO animals were treated once with $\mathrm{HBO}$ therapy $30 \mathrm{~min}$ after $\mathrm{ICH}$, with 2.5 ATA pressure and $100 \% \mathrm{O}_{2}$, boosting pressure for $15 \mathrm{~min}$, stable pressure for one hour and reducing pressure for $15 \mathrm{~min}$. Then they were sent to the hypobaric chamber (0.6 ATA) until 24 h after ICH. The HS and HI animals were always maintained in the hypobaric chamber for the end time point, and the animals in the PS and PI groups were only supplied air at normal pressure.

\section{Multimodal monitoring}

The $\mathrm{PbtO}_{2}$ probe (Lincox, Ratingen, Germany) and microdialysis (MD) probe (CMA, Kista, Sweden) were inserted and fixed firmly two hours after ICH. The positions of the $\mathrm{PbtO}_{2}$ probe and $\mathrm{MD}$ probe in the perihematomal tissue were confirmed via computed tomography, and these probes were very close to the hematoma (Fig. 1c). The data were collected one hour after insertion due to the unreliability of readings in the initial period of time postinsertion. Then, these parameters were continuously recorded from three to $12 \mathrm{~h}$ after $\mathrm{ICH}$. Microdialysates were perfused with a rate of $0.3 \mu \mathrm{l} / \mathrm{min}$ using an MD pump (CMA, Kista, Sweden), and samples were taken every hour. The MD vials were collected and immediately refrigerated at $-80{ }^{\circ} \mathrm{C}$. All vials were analyzed after the experiment using an MD automatic analyzer (CMA 600, Kista, Sweden).

\section{Neurobehavioral evaluation}

Behavior was assessed in each group $24 \mathrm{~h}$ after surgery using the Purdy neurological deficit scale with moderate modification [17]. The scale included tests of motor 
Table 1 Physiological parameters in minipigs after $\mathrm{ICH}$

\begin{tabular}{|c|c|c|c|c|c|c|}
\hline & Hours post-ICH & PS $(n=6)$ & $\mathrm{PI}(n=10)$ & $\mathrm{HS}(n=6)$ & $\mathrm{HI}(\mathrm{n}=10)$ & $\mathrm{HI}-\mathrm{HBO}(\mathrm{n}=10)$ \\
\hline \multirow[t]{3}{*}{ MAP (mmHg) } & 3 & $91.3 \pm 2.5$ & $93.8 \pm 2.1$ & $93.1 \pm 2.6$ & $94.2 \pm 2.3$ & $94.8 \pm 2.2$ \\
\hline & 6 & $91.4 \pm 2.4$ & $94.1 \pm 2.0$ & $92.2 \pm 2.6$ & $92.8 \pm 2.2$ & $94.9 \pm 2.2$ \\
\hline & 12 & $92.0 \pm 2.5$ & $93.0 \pm 1.9$ & $91.3 \pm 2.4$ & $93.7 \pm 2.2$ & $93.4 \pm 2.0$ \\
\hline \multirow[t]{3}{*}{ Plasma glucose (mM) } & 3 & $4.45 \pm 0.17$ & $4.55 \pm 0.13$ & $4.48 \pm 0.16$ & $4.41 \pm 0.12$ & $4.49 \pm 0.12$ \\
\hline & 6 & $4.55 \pm 0.17$ & $4.48 \pm 0.13$ & $4.46 \pm 0.15$ & $4.53 \pm 0.12$ & $4.48 \pm 0.12$ \\
\hline & 12 & $4.35 \pm 0.15$ & $4.27 \pm 0.12$ & $4.36 \pm 0.14$ & $4.21 \pm 0.11$ & $4.38 \pm 0.12$ \\
\hline \multirow[t]{3}{*}{ Core temperature $\left({ }^{\circ} \mathrm{C}\right)$} & 3 & $38.64 \pm 0.06$ & $38.73 \pm 0.06$ & $38.58 \pm 0.07$ & $38.70 \pm 0.06$ & $38.55 \pm 0.08$ \\
\hline & 6 & $38.58 \pm 0.06$ & $38.66 \pm 0.06$ & $38.53 \pm 0.06$ & $38.61 \pm 0.06$ & $38.48 \pm 0.07$ \\
\hline & 12 & $38.57 \pm 0.05$ & $38.60 \pm 0.06$ & $38.55 \pm 0.06$ & $38.53 \pm 0.05$ & $38.46 \pm 0.06$ \\
\hline \multirow[t]{3}{*}{$\mathrm{pH}$} & 3 & $7.37 \pm 0.02$ & $7.37 \pm 0.02$ & $7.46 \pm 0.03$ a,b & $7.47 \pm 0.02^{a, b}$ & $7.46 \pm 0.02^{a, b}$ \\
\hline & 6 & $7.38 \pm 0.02$ & $7.38 \pm 0.01$ & $7.46 \pm 0.03$ a,b & $7.45 \pm 0.02^{a, b}$ & $7.48 \pm 0.01 \mathrm{a}, \mathrm{b}$ \\
\hline & 12 & $7.38 \pm 0.02$ & $7.39 \pm 0.01$ & $7.47 \pm 0.03^{a, b}$ & $7.46 \pm 0.02^{a, b}$ & $7.47 \pm 0.02^{a, b}$ \\
\hline \multirow[t]{3}{*}{$\mathrm{HCO}_{3}^{-}(\mathrm{mM})$} & 3 & $24.50 \pm 1.31$ & $21.82 \pm 1.09$ & $18.18 \pm 0.87^{\mathrm{a}, \mathrm{b}}$ & $18.53 \pm 0.65^{a, b}$ & $18.46 \pm 0.66^{a, b}$ \\
\hline & 6 & $24.92 \pm 1.27$ & $22.03 \pm 1.19$ & $18.46 \pm 0.84^{a, b}$ & $17.31 \pm 0.76^{a, b}$ & $18.85 \pm 0.62^{a, b}$ \\
\hline & 12 & $23.87 \pm 1.32$ & $22.57 \pm 1.17$ & $17.97 \pm 0.91 \mathrm{a}, \mathrm{b}$ & $17.73 \pm 0.67^{\mathrm{a}, \mathrm{b}}$ & $18.64 \pm 0.71 \mathrm{a}, \mathrm{b}$ \\
\hline \multirow[t]{3}{*}{$\mathrm{SaO} 2$} & 3 & $97.1 \pm 0.5$ & $96.4 \pm 0.4$ & $86.7 \pm 0.9^{a, b}$ & $86.4 \pm 0.6^{a, b}$ & $87.0 \pm 0.7^{a, b}$ \\
\hline & 6 & $97.8 \pm 0.6$ & $96.7 \pm 0.5$ & $87.3 \pm 0.8^{a, b}$ & $85.9 \pm 0.7^{a, b}$ & $86.8 \pm 0.7^{a, b}$ \\
\hline & 12 & $97.5 \pm 0.6$ & $97.1 \pm 0.5$ & $87.2 \pm 0.8^{a, b}$ & $86.5 \pm 0.6^{a, b}$ & $86.7 \pm 0.6^{a, b}$ \\
\hline \multirow[t]{3}{*}{$\mathrm{PaO}_{2}(\mathrm{mmHg})$} & 3 & $89.7 \pm 1.5$ & $84.5 \pm 1.2$ & $67.3 \pm 1.4^{\mathrm{a}, \mathrm{b}}$ & $63.1 \pm 0.9^{a, b}$ & $64.3 \pm 1.0^{a, b}$ \\
\hline & 6 & $90.7 \pm 1.8$ & $85.6 \pm 1.1$ & $68.3 \pm 1.5^{a, b}$ & $63.6 \pm 1.0^{a, b}$ & $63.9 \pm 1.0 \mathrm{a}, \mathrm{b}$ \\
\hline & 12 & $88.8 \pm 1.5$ & $88.4 \pm 1.2$ & $68.8 \pm 1.5^{a, b}$ & $65.3 \pm 1.0^{a, b}$ & $66.3 \pm 1.2^{a, b}$ \\
\hline \multirow[t]{3}{*}{$\mathrm{PaCO}_{2}(\mathrm{mmHg})$} & 3 & $35.1 \pm 1.0$ & $32.9 \pm 0.6$ & $25.1 \pm 0.6^{a, b}$ & $23.5 \pm 0.5^{a, b}$ & $24.8 \pm 0.5^{a, b}$ \\
\hline & 6 & $34.5 \pm 0.9$ & $33.8 \pm 0.6$ & $24.9 \pm 0.6^{a, b}$ & $24.4 \pm 0.6^{a, b}$ & $25.6 \pm 0.6^{a, b}$ \\
\hline & 12 & $33.9 \pm 0.8$ & $34.2 \pm 0.7$ & $25.4 \pm 0.7^{a, b}$ & $24.1 \pm 0.6^{a, b}$ & $25.1 \pm 0.6^{a, b}$ \\
\hline
\end{tabular}

At each time point examined, plasma glucose, MAP and core temperature showed no significant differences between the five groups. Values of blood gas analysis showed significant differences between the plain (low altitude) and high-altitude animals. There was no significant difference between the plain groups, and between the high-altitude groups. HI: high altitude blood infusion group, HI-HBO: high-altitude blood infusion plus HBO therapy group, HS: high altitude sham operation group, MAP: mean arterial blood pressure, PI: plain blood infusion group, PS: plain sham operation group

${ }^{\mathrm{a}} \mathrm{P}<0.05$, compared with PS; ${ }^{\mathrm{b}} \mathrm{P}<0.05$, compared with $\mathrm{PI}$

function (0 to 5), consciousness (0 to 4$)$, head turning (0 to 1$)$, circling (0 to 1$)$ and hemianopsia (0 to 1$)$. Neurobehavioral evaluation was graded on a scale of 0 to 12 (completely normal score, 0 ; maximal deficit score, 12). Tests were conducted by an observer blinded to the groups.

\section{Brain tissue water content}

At $24 \mathrm{~h}$ after surgery, half of the animals in each group were deeply anesthetized, and their skull was opened. Two brain tissue samples around the hematoma of each brain were cut and weighed. These samples were put into an oven at $100{ }^{\circ} \mathrm{C}$ for $72 \mathrm{~h}$ until a constant weight was reached. Water contents were expressed as a percentage of wet weight.

\section{Nissl staining}

The other half of the animals of each group were anesthetized, and their brains were perfused in situ with $10 \%$ formalin. Then, the brains were soaked in $4 \%$ paraformaldehyde solution at $4{ }^{\circ} \mathrm{C}$ for $72 \mathrm{~h}$. Brain tissue adjacent to the hematoma was cut, embedded in $30 \%$ paraffin and cut into $20-\mu \mathrm{m}$ thick sections. These sections were deparaffinized with xylene and graded alcohol. Toluidine blue was used according to a standard protocol. Each section was observed and photographed under an optical microscope (Olympus, Tokyo, Japan).

\section{Terminal deoxynucleotidyl transferase dUTP nick-end labeling staining}

The terminal deoxynucleotidyl transferase dUTP nickend labeling (TUNEL) assay was performed using the In situ Cell Death Detection Kit (Roche Molecular Biochemicals, Mannheim, Germany) that labels DNA strand breaks with fluorescein isothiocyanate according to a standard protocol. Each section was observed and photographed under a confocal microscope (Carl Zeiss, Oberkochen, Germany). Negative controls were obtained by omitting the TdT enzyme. The percentage of 
TUNEL-positive cells was expressed as the number of TUNEL-stained nuclei divided by the total number of 4',6-diamidino-2-phenylindole (DAPI)-stained nuclei.

\section{Statistical analysis}

All data were expressed as the means \pm SEM. A model of repeated-measures analysis of variance (ANOVA) with post-hoc Bonferroni correction was used for analysis of various physiological parameters. One-way ANOVA with post-hoc Fisher's least significant difference test was used for neurological scores and brain edema. All analyses were calculated using PASW software version 18 (SPSS, Chicago, USA). Statistical significance was preset at $P<0.05$.

\section{Results}

\section{Tissue oxygenation in the perihematomal tissue}

All minipigs survived and were observed to the end of the experiment. Although $\mathrm{PbtO}_{2}$ in the $\mathrm{HS}$ animals was lower than in the PS animals at most of the time points, no significant difference existed between these two groups $(P>0.05)$. At each time point, $\mathrm{PbtO}_{2}$ in the $\mathrm{PI}, \mathrm{HI}$ and $\mathrm{HI}-\mathrm{HBO}$ animals was significantly lower than in the PS and HS animals. Furthermore, $\mathrm{PbtO}_{2}$ in the $\mathrm{HI}$ animals was significantly lower than in the PI animals and the HI-HBO animals at each time point $(P<0.05)$. However, there were no significant differences between the PI and HI-HBO animals at any time point. $\mathrm{PbtO}_{2}$ at 11 or $12 \mathrm{~h}$ was significantly higher than at three hours in the HI-HBO animals (Fig. 2a).

\section{Cerebral metabolism in the perihematomal tissue}

There was no significant difference between the PS and HS animals in glucose, lactate, lactate/pyruvate ratio $(\mathrm{LPR})$ and glutamate $(P>0.05)$. At each time point, glucose in the PI, HI and HI-HBO animals was significantly lower than in the PS and HS animals $(P<0.05)$. Although glucose in the HI animals was lower than in the PI and HI-HBO animals at most of the time points, no significant difference was observed among the three groups (Fig. 2b). Lactate was significantly higher at five or six hours than at $12 \mathrm{~h}$ in the HI animals, and it was significantly higher at six hours than at 11 or $12 \mathrm{~h}$ in the HI$\mathrm{HBO}$ animals. At each time point, lactate in the PI, HI and $\mathrm{HI}-\mathrm{HBO}$ animals was significantly higher than in the PS and HS animals $(P<0.05)$. Although lactate in the HI animals was higher than in the PI and HI-HBO animals at most of the time points, there was no significant difference among the three groups (Fig. 2c). It was significantly higher at six hours than at $12 \mathrm{~h}$ in the $\mathrm{HI}$ and HI-HBO animals about LPR. At each time point, LPR in the PI, HI and $\mathrm{HI}-\mathrm{HBO}$ animals was significantly higher than in the PS and HS animals $(P<0.05)$. Furthermore, LPR in the HI animals was significantly higher than in the PI and HI-
HBO animals $(P<0.05)$. However, there was no significant difference between the PI and HI-HBO animals (Fig. 2d). Glutamate was significantly higher at four hours than at $12 \mathrm{~h}$ in the HI and HI-HBO animals. It was also significantly higher in the PI, HI and HI-HBO animals than in the PS and HS animals $(P<0.05)$. Furthermore, it was significantly higher in the $\mathrm{HI}$ animals than in the PI and HI$\mathrm{HBO}$ animals at each time point $(P<0.05)$. No significant difference existed in the PI and HI-HBO animals (Fig. 2e).

\section{Neurological deficits and brain edema}

There was no significant difference between the PS and HS animals in neurological scores or brain water content. High-altitude ICH showed more serious impairments in neurological functioning than low-altitude $\mathrm{ICH}$ $(P<0.05)$. Further, there was significant improvement in neurological deficits after $\mathrm{HBO}$ treatment at $24 \mathrm{~h}$ postinjury (Fig. 3a). High-altitude ICH also showed higher brain water content than low-altitude ICH $(P<0.05)$. After HBO treatment, brain water content was significantly decreased $24 \mathrm{~h}$ after ICH (Fig. 3b).

\section{DNA damage and neuronal damage in perihematomal tissue}

At $24 \mathrm{~h}$ post-ICH, coronal brain slices showed ovalshaped hematomas at the right basal ganglia (Fig. 4a). PS and HS animals showed lots of stained neurons with normal structure in Nissl staining. A decrease in the number of stained cells, as well as unclear or damaged structure in most of the neurons around the hematoma, were shown in PI animals. Furthermore, the decrease in the number of stained cells and structural damage of the neurons was more severe in the HI animals when compared with the PI animals. However, the neuronal damage in the HI-HBO group was less than that in the HI group (Fig. 4b). In addition, similar results for DNA damage were found with TUNEL staining. TUNELpositive cells were evident in the perihematomal tissue at $24 \mathrm{~h}$ post-ICH. The number of TUNEL-positive cells around the hematoma increased dramatically in the HI group compared with the PI group $(P<0.05)$. Significantly fewer TUNEL-positive cells were found in the HIHBO group (Fig. 4c).

\section{Discussion}

High-altitude ICH has been a somewhat obscure clinical problem to date, and there is a severe lack of experimental animal research into high-altitude $\mathrm{ICH}$. In this animal study, high-altitude ICH animals manifested lower $\mathrm{PbtO}_{2}$, higher levels of LPR and glutamate, and more severe brain lesion and neurological outcomes than lowaltitude ICH. HBO treatment (2.5 ATA for $60 \mathrm{~min}$ ) 30 min after high-altitude ICH improved cerebral physiology, pathological damage and neurological deficit. One 


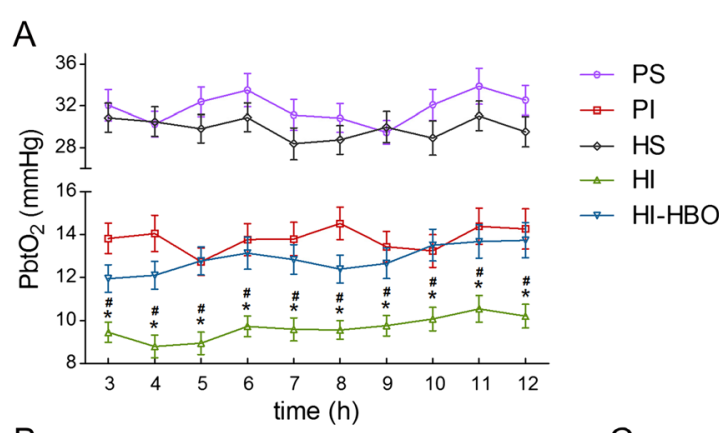

$\mathrm{B}$



D



C

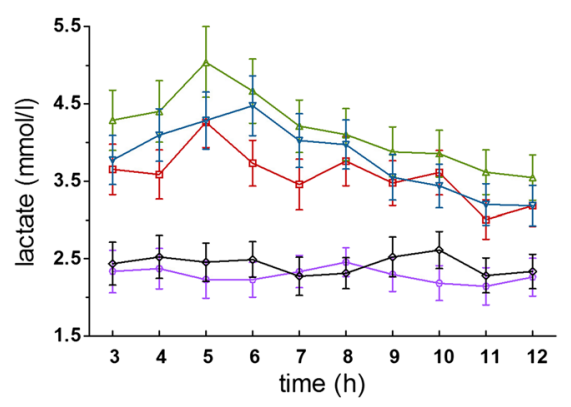

E

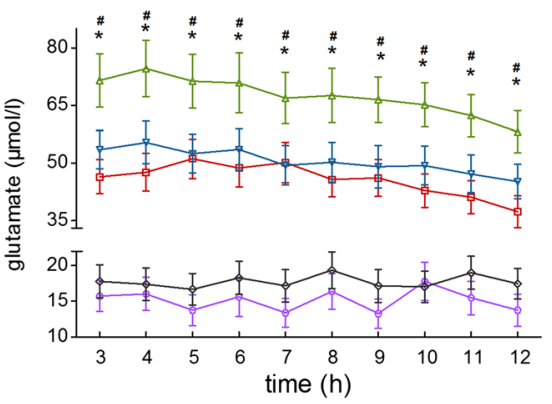

Fig. 2 Changes in cerebral oxygenation and metabolism from three to $12 \mathrm{~h}$ post-ICH. a At each time point, $\mathrm{PbtO}_{2}$ in $\mathrm{HI}$ animals was significantly lower than in $\mathrm{Pl}$ animals, and it was significantly elevated in $\mathrm{HI}-\mathrm{HBO}$ animals. b Although the level of glucose in $\mathrm{HI}$ animals was lower than in the $\mathrm{PI}$ or HI-HBO animals at most of the time points, there was no significant difference between the three groups. c Likewise, lactate in HI animals was higher than in the PI or HI-HBO animals at all time points, but no significant difference existed between them. $\mathbf{d}$ LPR in HI animals was significantly higher than in the $\mathrm{PI}$ and $\mathrm{HI}-\mathrm{HBO}$ animals at each time point. e Likewise, glutamate in $\mathrm{HI}$ animals was significantly higher than in the $\mathrm{PI}$ and $\mathrm{HI}-\mathrm{HBO}$ animals at each time point. ${ }^{\#} P<0.05$, compared with $\mathrm{Pl} ;{ }^{*} P<0.05$, compared with $\mathrm{HI}-\mathrm{HBO}$


Fig. 3 Changes in neurological functioning and brain edema $24 \mathrm{~h}$ after ICH. a High-altitude ICH showed more serious neurological function impairments than low-altitude ICH. However, there was no significant difference between $\mathrm{HI}$ and $\mathrm{HI}-\mathrm{HBO}$ animals. b High-altitude $\mathrm{ICH}$ also showed more serious brain edema than low-altitude ICH. Moreover, brain water content in HI-HBO animals significantly decreased. ${ }^{*} P<0.05$ 




Fig. 4 Nissl and TUNEL staining of the perihematomal tissue $24 \mathrm{~h}$ after $\mathrm{ICH}$. a Coronal brain slice shows oval-shaped hematomas at the right basal ganglia. $\mathbf{b}$ Nissl staining showing a decrease in the number of stained cells and more severe structural damage of the neurons around the hematoma in $\mathrm{HI}$ animals. However, the neuronal damage in $\mathrm{HI}-\mathrm{HBO}$ animals was reduced. Right arrow indicates normal neuron, up arrow indicates damaged neuron in the photos. c Quantification analysis indicating TUNEL-positive cells increased in $\mathrm{HI}$ animals and decreased in $\mathrm{HI}-\mathrm{HBO}$ animals. The percentage of TUNEL-positive cells was expressed as the number of TUNEL-stained nuclei divided by the total number of DAPI-stained nuclei. ${ }^{*} P<0.05$. Bar $=100 \mu m$

possible pathophysiological mechanism whereby $\mathrm{HBO}$ treatment attenuates brain injury is the modulation of cerebral oxygenation and metabolism during experimental high-altitude $\mathrm{ICH}$.

$\mathrm{PbtO}_{2}$, the partial pressure of oxygen in the extracellular fluid of the brain tissue, reflects the availability of oxygen for oxidative energy production. Although $\mathrm{PbtO}_{2}$ does not directly assess cerebral blood flow, it is a complex and dynamic parameter representing the interaction between the delivery and demand of brain oxygen, as well as oxygen diffusion gradients [11]. $\mathrm{PbtO}_{2}$ monitoring can be used to identify cerebral perfusion pressure targets for optimal brain tissue oxygenation, and low perihematomal $\mathrm{PbtO}_{2}$ correlates with poorer outcomes after $\mathrm{ICH}$ [10]. In this study, high-altitude $\mathrm{ICH}$ showed lower $\mathrm{PbtO}_{2}$ than low-altitude $\mathrm{ICH}$ in the acute stage, and no significant difference in MAP existed between these groups. In addition to MAP, changes in arterial oxygenation can influence $\mathrm{PbtO}_{2}$ following $\mathrm{ICH}$ [18]. Therefore, the low $\mathrm{PaO}_{2}$ in response to hypobaric hypoxia may be an important cause of the worse cerebral oxygenation post$\mathrm{ICH}$. The observation that lower $\mathrm{PbtO}_{2}$ occurred in animals exposed to hypobaric hypoxia rather than normoxia after head trauma was consistent with our findings [19].

Despite some indicators of hypoperfusion, true ischemia in the perihematomal region was difficult to prove because of disproportionate reductions in the cerebral metabolic rate for oxygen and preserved oxygen extraction fractions in the perihematomal tissue [20]. A switch of concept from ischemic to metabolic penumbra was suggested recently. The transient focal increases in glucose metabolism have been interpreted as signs of ongoing neuronal injury in the metabolic penumbral tissue [21]. One or more non-ischemic mechanisms may contribute to this change in energy metabolism, including neuroinflammation, cytotoxicity or seizure activity. The interstitial glucose concentration is attributed to the balance between delivery from the blood capillaries and the cellular uptake. A low level of glucose was associated with worse outcome after brain injury [10]. In this study, a decrease in interstitial glucose concentration in the early phase, which was interpreted as an increase in glucose consumption, was observed after ICH. However, hypobaric hypoxia did not result in markedly lower glucose than normoxia post-ICH. One reason may be that hypoxia induces an increase in cerebral glucose concentrations in response to increased glucose transporters in brain microvessels and blood-to-brain glucose influx [22]. Another may be regional-specific changes in the cerebral glucose metabolism exposed to hypobaric hypoxia [23, 24]. Additionally, the cerebral glucose consumption may reach maximal capacity, so the glucose level is maintained at its lowest level possible. Serum glucose levels can affect cerebral glucose metabolism, so systemic glucose variability may be associated with poor outcome in critically ill patients [25]. However, 
interference in cerebral metabolism from serum glucose was low in this study due to the similar levels of serum glucose in each group.

In addition to reduced extracellular glucose, elevated lactate is also observed in some brain injury studies [26]. Additionally, lactate accumulations or pyruvate decreases in the extracellular space lead to an increase in LPR, which reflects the oxidative metabolism within the cytoplasm. In this study, cerebral lactate and LPR increased and were sustained until the end time point. Furthermore, patients after surgical evacuation of a hematoma with a poor outcome had significantly higher lactate and LPR than patients with a good outcome [27]. Although cerebral lactate may increase at high altitude $[28,29]$, lactate did not dramatically elevate after ICH at high altitude compared to low altitude in our study. We speculate that lactate may be used as an alternate energy source for neurons, potentially via shuttling of glycolytically derived carbons from astrocytes, especially in hypoxia [30]. Another ICH study reported that LPR and intracranial pressure decreased after mannitol administration despite a lack of increase in MD glucose, pyruvate and $\mathrm{PbtO}_{2}$ [31]. Recently, LPR has been interpreted as energy metabolic distress in the absence of ischemia, which possibly results from mitochondrial dysfunction, seizures or reduced substrate availability. In the present study, larger increases in LPR induced by hypobaric hypoxia indicate worse energy metabolism dysfunction at high altitude than at low altitude in the early stages post-ICH. Increased LPR in the bronchoalveolar fluid was also observed in high-altitude lung injury [32].

Elevated glutamate levels in the perihematomal region after ICH have been demonstrated in some low-altitude studies [33], and our data of glutamate are consistent with this finding. Glutamate activation of NMDA or AMPA receptors can result in increased glucose metabolism in perihematomal tissue after ICH [34]. Furthermore, our results suggest a higher accumulation of glutamate around the hematoma soon after high-altitude $\mathrm{ICH}$. The expression change in glutamate receptors, such as NR1 and GluR2, mediates glutamate excitotoxicity in chronic hypobaric hypoxia [35]. These observations of multi-parameters show the complex features of brain metabolism as a function of substrate delivery, transport and consumption in the variable demand of the injured brain, especially at high altitude. Any interpretation of one single factor is limited by virtue of its intricate nature. Thus, more severe brain oxygenation reduction, energy metabolism dysfunction and glutamate accumulation occur jointly in the early stages of highaltitude ICH characterized by hypobaric hypoxia. Direct or indirect actions of cerebral oxygenation and energy metabolism distress, as well as glutamate excitotoxicity, synergistically elicit the death and degeneration of neural cells, which aggravates brain edema and cerebral damage after high-altitude $\mathrm{ICH}$.

$\mathrm{HBO}$ has been widely used as the primary therapy in patients with decompression sickness, carbon monoxide poisoning and arterial gas embolism. Plenty of studies suggest that $\mathrm{HBO}$ treatment is effective in brain injury at low altitude. Early HBO therapy mitigates bloodbrain barrier disruption and suppresses the progression of brain edema post-ICH by preventing occludin degradation and matrix metalloproteinase- 9 activation in the perihematomal tissue [36]. In a murine ischemic stroke study, altered striatal energy metabolites and glutamate can be regulated by $\mathrm{HBO}$, which might contribute to the neuroprotection of HBO therapy [37]. Additionally, normobaric hyperoxia also increases brain tissue oximetry, with a variable effect on lactate and LPR in severe traumatic brain injury patients [38]. Another study reported that $\mathrm{HBO}$ improved $\mathrm{PbtO}_{2}$ and metabolic distress, and had a more robust post-treatment effect than normobaric hyperoxia on oxidative cerebral metabolism, related to its ability to produce a $\mathrm{PbtO}_{2}$ of $200 \mathrm{mmHg}$ or higher [13]. After early $\mathrm{HBO}$ treatment, an increase in $\mathrm{PbtO}_{2}$ may improve cerebral metabolism, cerebrovascular autoregulation, cerebral blood flow, neuroinflammation and brain edema. In turn, improvements in these can raise cerebral oxygenation. In this study, early HBO therapy also markedly elevated $\mathrm{PbtO}_{2}$ for $12 \mathrm{~h}$ in hypobaric hypoxia. A study about high-altitude brain injury also reported that $\mathrm{HBO}$ treatment can increase $\mathrm{PbtO}_{2}$ and regional cerebral blood flow $24 \mathrm{~h}$ after brain injury and benefit recovery [19]. HBO treatment following highaltitude ICH alleviated secondary brain injury and neurological deficits, which was attributed to ameliorated brain tissue oxygenation, energy metabolism dysfunction and glutamate excitotoxicity. HBO therapy has also been applied in many other diseases at high altitude. One hour of treatment in a portable hyperbaric chamber leads to a short-term improvement in symptoms of acute mountain sickness [39]. Improvements in the focal oxygen supply by $\mathrm{HBO}$ ameliorated calcific uremic arteriolopathy in high-altitude areas [40]. Additionally, high-altitude pulmonary edema and lung injury can be reduced by an induction of heat-shock protein 70 in the lung in response to HBO preconditioning [41].

There are some limitations to this study. First, maintenance in a hypobaric chamber for three days may be too short to totally emulate the long duration of $\mathrm{ICH}$ formation at high altitude. However, it must be noted that the current study was intended to be a hypothesisgenerating pilot study. The absence of intracranial pressure and cerebral perfusion pressure monitoring is another limitation. Additionally, because our autologous blood injection model does not mimic small vessel rupture in $\mathrm{ICH}$, microvascular breakdown effects are 
difficult to assess. Furthermore, the size of the sample is small and a larger number is needed for further investigation.

\section{Conclusions}

Our findings indicate more serious disturbance of tissue oxygenation and cerebral metabolism, as well as worse brain pathology and neurological outcomes, in highaltitude ICH in comparison to low-altitude $\mathrm{ICH}$ in the acute stages in our porcine model. Early HBO treatment reduced brain edema and tissue damage via amelioration of cerebral oxygenation and metabolism, and this may be an important mechanism whereby $\mathrm{HBO}$ attenuates secondary brain injury following high-altitude ICH. All the data support modulation of tissue oxygenation and cerebral metabolism in perihematomal tissue as a potential therapeutic target, and suggest that early $\mathrm{HBO}$ treatment may be beneficial for ICH at high altitude.

\section{Key messages}

- High-altitude ICH shows more serious disturbance of tissue oxygenation and cerebral metabolism in comparison to low-altitude ICH in the acute stages.

- Early HBO treatment reduced acute brain injury through a mechanism involving the amelioration of the derangement of cerebral oxygenation and metabolism following high-altitude $\mathrm{ICH}$.

\section{Abbreviations}

ATA: absolute atmosphere; HBO: hyperbaric oxygen; HI: high altitude blood infusion group; HI-HBO: high-altitude blood infusion plus; HBO: therapy group; HS: high altitude sham operation group; ICH: Intracerebral hemorrhage; LPR: lactate/pyruvate ratio; MAP: mean arterial pressure; MD: microdialysis; PbtO2: brain tissue oxygen partial pressure; PI: plain blood infusion group; PS: plain sham operation group.
\end{abstract}

\section{Competing interests}

The authors have not disclosed any potential conflicts of interest.

\section{Authors' contributions}

HTZ and CB carried out the neuromonitoring and morphological studies, and drafted the manuscript. XJL and WL carried out behavioral studies. JCY performed the statistical analysis. HF and GZ participated in the design of the study, and helped to revise the manuscript. LJK conceived of the study, participated in its design and coordination, and helped to draft the manuscript. All authors read and approved the final manuscript.

\section{Acknowledgements}

This work was supported, in part, by grants from by the National '973' Project of China (grant number: 2014CB541605), Sci \& Tech Research Project of PLA, China (grant number: 08G087) and the National Science Foundation of China (grant numbers: 81401012, 81070979).

\section{Author details}

'Department of Neurosurgery, Southwest Hospital, Third Military Medical University, 30 Gaotanyan Street, Chongqing 400038, China. ${ }^{2}$ Department of Military Psychology, College of Psychology, Third Military Medical University, 30 Gaotanyan Street, Chongqing 400038, China.

Received: 20 February 2015 Accepted: 4 June 2015

Published online: 15 June 2015

\section{References}

1. van Asch CJ, Luitse MJ, Rinkel GJ, van der Tweel I, Algra A, Klijn CJ. Incidence, case fatality, and functional outcome of intracerebral haemorrhage over time, according to age, sex, and ethnic origin: a systematic review and meta-analysis. Lancet Neurol. 2010;9:167-76.

2. Adeoye O, Broderick JP. Advances in the management of intracerebral hemorrhage. Nat Rev Neurol. 2010;6:593-601.

3. Basnyat $B, W u T$, Gertsch JH. Neurological conditions at altitude that fall outside the usual definition of altitude sickness. High Alt Med Biol. 2004:5:171-9.

4. Jha SK, Anand AC, Sharma V, Kumar N, Adya CM. Stroke at high altitude: Indian experience. High Alt Med Biol. 2002:3:21-7.

5. Jaillard AS, Hommel M, Mazetti P. Prevalence of stroke at high altitude (3380 m) in Cuzco, a town of Peru. A population-based study. Stroke. 1995;26:562-8

6. Zhao Y, Yao Z, D'Souza W, Zhu C, Chun H, Zhuoga C, et al. An epidemiological survey of stroke in Lhasa, Tibet. China Stroke. 2010;41:2739-43.

7. Fang J, Zhuo-Ga C, Zhao Y, Kong F, Si Y, Liu M, et al. Characteristics of stroke in Tibet autonomous region in China: a hospital-based study of acute stroke. Eur Neurol. 2011;66:151-8.

8. Bhatia A, Gupta AK. Neuromonitoring in the intensive care unit. II. Cerebral oxygenation monitoring and microdialysis. Intensive Care Med. 2007;33:1322-8

9. Helbok R, Schiefecker AJ, Beer R, Dietmann A, Antunes AP, Sohm F, et al. Early brain injury after aneurysmal subarachnoid hemorrhage: a multimodal neuromonitoring study. Crit Care. 2015;19:75.

10. Ko SB, Choi HA, Parikh G, Helbok R, Schmidt JM, Lee K, et al. Multimodality monitoring for cerebral perfusion pressure optimization in comatose patients with intracerebral hemorrhage. Stroke. 2011;42:3087-92.

11. Kirkman MA, Smith M. Supratentorial intracerebral hemorrhage: a review of the underlying pathophysiology and its relevance for multimodality neuromonitoring in neurointensive care. J Neurosurg Anesthesiol. 2013;25:228-39.

12. Qin Z, Karabiyikoglu M, Hua Y, Silbergleit R, He Y, Keep RF, et al. Hyperbaric oxygen-induced attenuation of hemorrhagic transformation after experimental focal transient cerebral ischemia. Stroke. 2007:38:1362-7.

13. Rockswold SB, Rockswold GL, Zaun DA, Zhang X, Cerra CE, Bergman TA, et al. A prospective, randomized clinical trial to compare the effect of hyperbaric to normobaric hyperoxia on cerebral metabolism, intracranial pressure, and oxygen toxicity in severe traumatic brain injury. J Neurosurg. 2010;112:1080-94

14. Niaz A, Nayyar S. Cerebrovascular stroke at high altitude. J Coll Physicians Surg Pak. 2003;13:446-8.

15. Zhu H, Li F, Zou M, Xue X, Yuan J, Feng H, et al. Experimental high-altitude intracerebral hemorrhage in minipigs: histology, behavior, and intracranial pressure in a double-injection model. Acta Neurochir (Wien). 2013;155:655-61.

16. Gu Y, Hua Y, Keep RF, Morgenstern LB, Xi G. Deferoxamine reduces intracerebral hematoma-induced iron accumulation and neuronal death in piglets. Stroke. 2009;40:2241-3.

17. Purdy PD, Devous Sr MD, Batjer HH, White 3rd CL, Meyer Y, Samson DS. Microfibrillar collagen model of canine cerebral infarction. Stroke. 1989;20:1361-7.

18. Hemphill 3rd JC, Morabito D, Farrant M, Manley GT. Brain tissue oxygen monitoring in intracerebral hemorrhage. Neurocrit Care. 2005;3:260-70.

19. Hu S, Li F, Luo H, Xia Y, Zhang J, Hu R, et al. Amelioration of rCBF and $\mathrm{PbtO} 2$ following $\mathrm{TBI}$ at high altitude by hyperbaric oxygen pre-conditioning. Neurol Res. 2010;32:173-8.

20. Vespa PM. Metabolic penumbra in intracerebral hemorrhage. Stroke. 2009:40:1547-8

21. Zazulia AR, Videen TO, Powers WJ. Transient focal increase in perihematomal glucose metabolism after acute human intracerebral hemorrhage. Stroke. 2009;40:1638-43.

22. Harik SI, Lust WD, Jones SC, Lauro KL, Pundik S, LaManna JC. Brain glucose metabolism in hypobaric hypoxia. J Appl Physiol (1985). 1995;79:136-40.

23. Merz TM, Treyer V, Hefti U, Spengler CM, Schwarz U, Buck A, et al. Changes in cerebral glucose metabolism after an expedition to high altitudes. High Alt Med Biol. 2006;7:28-38.

24. Hochachka PW, Clark CM, Matheson GO, Brown WD, Stone CK, Nickles RJ, et al. Effects on regional brain metabolism of high-altitude hypoxia: a study of six US marines. Am J Physiol. 1999;277:R314-9. 
25. Kurtz P, Claassen J, Helbok R, Schmidt J, Fernandez L, Presciutti M, et al. Systemic glucose variability predicts cerebral metabolic distress and mortality after subarachnoid hemorrhage: a retrospective observational study. Crit Care. 2014;18:R89.

26. Timofeev I, Carpenter KL, Nortje J, Al-Rawi PG, O'Connell MT, Czosnyka M, et al. Cerebral extracellular chemistry and outcome following traumatic brain injury: a microdialysis study of 223 patients. Brain. 2011;134:484-94.

27. Wang $\mathrm{E}, \mathrm{Ho} \mathrm{CL}$, Lee $\mathrm{KK}, \mathrm{Ng} \mathrm{I}$, Ang BT. Changes in brain biochemistry and oxygenation in the zone surrounding primary intracerebral hemorrhage. Acta Neurochir Suppl. 2008;102:293-7.

28. Duffy TE, Nelson SR, Lowry OH. Cerebral carbohydrate metabolism during acute hypoxia and recovery. J Neurochem. 1972;19:959-77.

29. Shrivastava K, Ram MS, Bansal A, Singh SS, llavazhagan G. Cobalt supplementation promotes hypoxic tolerance and facilitates acclimatization to hypobaric hypoxia in rat brain. High Alt Med Biol. 2008;9:63-75.

30. Cater HL, Chandratheva A, Benham CD, Morrison 3rd B, Sundstrom LE. Lactate and glucose as energy substrates during, and after, oxygen deprivation in rat hippocampal acute and cultured slices. J Neurochem. 2003;87:1381-90.

31. Helbok R, Kurtz P, Schmidt JM, Stuart RM, Fernandez L, Malhotra R, et al. Effect of mannitol on brain metabolism and tissue oxygenation in severe haemorrhagic stroke. J Neurol Neurosurg Psychiatry. 2011;82:378-83.

32. Lin HJ, Wang CT, Niu KC, Gao C, Li Z, Lin MT, et al. Hypobaric hypoxia preconditioning attenuates acute lung injury during high-altitude exposure in rats via up-regulating heat-shock protein 70. Clin Sci (Lond). 2011;121:223-31.

33. Qureshi Al, Ali Z, Suri MF, Shuaib A, Baker G, Todd K, et al. Extracellular glutamate and other amino acids in experimental intracerebral hemorrhage: an in vivo microdialysis study. Crit Care Med. 2003;31:1482-9.

34. Sharp F, Liu DZ, Zhan X, Ander BP. Intracerebral hemorrhage injury mechanisms: glutamate neurotoxicity, thrombin, and Src. Acta Neurochir Suppl. 2008;105:43-6.

35. Hota SK, Barhwal K, Singh SB, Sairam M, llavazhagan G. NR1 and GluR2 expression mediates excitotoxicity in chronic hypobaric hypoxia. J Neurosci Res. 2008:86:1142-52.

36. Zhou W, Marinescu M, Veltkamp R. Only very early oxygen therapy attenuates posthemorrhagic edema formation and blood-brain barrier disruption in murine intracerebral hemorrhage. Neurocrit Care. 2015;22:121-32.

37. Badr AE, Yin W, Mychaskiw G, Zhang JH. Effect of hyperbaric oxygen on striatal metabolites: a microdialysis study in awake freely moving rats after MCA occlusion. Brain Res. 2001;916:85-90.

38. Nortje J, Coles JP, Timofeev I, Fryer TD, Aigbirhio Fl, Smielewski P, et al. Effect of hyperoxia on regional oxygenation and metabolism after severe traumatic brain injury: preliminary findings. Crit Care Med. 2008;36:273-81.

39. Bartsch P, Merki B, Hofstetter D, Maggiorini M, Kayser B, Oelz O. Treatment of acute mountain sickness by simulated descent: a randomised controlled trial. BMJ. 1993;306:1098-101.

40. Deng Y, Xie G, Li C, Zhang H, Yang B, Chen X, et al. Calcific uremic arteriolopathy ameliorated by hyperbaric oxygen therapy in high-altitude area. Ren Fail. 2014;36:1139-41.

41. Tsai MC, Lin HJ, Lin MT, Niu KC, Chang CP, Tsao TC. High-altitude pulmonary edema can be prevented by heat shock protein 70-mediated hyperbaric oxygen preconditioning. J Trauma Acute Care Surg. 2014;77:585-91.

\section{Submit your next manuscript to BioMed Central and take full advantage of:}

- Convenient online submission

- Thorough peer review

- No space constraints or color figure charges

- Immediate publication on acceptance

- Inclusion in PubMed, CAS, Scopus and Google Scholar

- Research which is freely available for redistribution 